\title{
Condições de trabalho em uma madeireira em Belo Jardim, Pernambuco
}

\author{
Luana Guedes da Silva CAVALCANTI ${ }^{2}$, Rafael Leite BRAZ ${ }^{2 *}$, \\ Eliane Cristina Sampaio de FREITAS ${ }^{2}$, Anderson Oliveira de LIMA ${ }^{2}$ \\ ${ }^{1}$ Trabalho selecionado no VI Congresso Nordestino de Engenharia Florestal, Mossoró, RN, Brasil. \\ ${ }^{2}$ Universidade Federal Rural de Pernambuco, Recife, Pernambuco, Brasil. \\ *E-mail: rlbraz.ufrpe@gmail.com
}

Recebido em maio/2019; Aceito em junho/2019.

\begin{abstract}
RESUMO: O setor madeireiro, apesar de possuir destaque dentro do cenário industrial brasileiro, caracteriza-se como um setor com grande potencial de ocorrência de acidentes e desencadeamento de doenças ocupacionais, mostrando que este não gera impacto somente na natureza, mas na qualidade de vida. O objetivo desse trabalho foi identificar as condições de trabalho em uma indústria de beneficiamento de madeira em Belo Jardim, Pernambuco, sob o ponto de vista da salubridade e da segurança, a fim de propor melhorias e adequações a serem realizadas de acordo com a legislação trabalhista vigente. A partir das metodologias das Normas de Higiene Ocupacional, verificou-se a necessidade de mudanças pontuais no layout da empresa, além da necessidade de trocar o protetor auricular e desenvolver um projeto para otimizar a iluminação da fábrica de maneira geral. Para atendimento à legislação trabalhista, existe a necessidade de elaboração de laudo técnico de condições ambientais do trabalho, formação de comissão interna de prevenção de acidentes e desenvolvimento de um programa de controle médico e saúde ocupacional, incentivando a prática de realização de exames periódicos nos funcionários. Palavras-chave: salubridade; ergonomia; segurança do trabalho; legislação trabalhista.
\end{abstract}

\section{Working conditions in a wood industry in Belo Jardim, Pernambuco}

\begin{abstract}
The timber industry in general has a great prominence within the Brazilian industrial scenario, is still a sector with great potential for accidents and triggering of occupational diseases, showing that this does not only impact on nature, but on the quality of life This study aimed to identify the working conditions in a wood processing industry in Belo Jardim, Pernambuco, from the point of view of health and safety, in order to adapt them according to the current labor legislation. Based on the methodologies of Occupational Hygiene Standards, it was verified the need for specific changes in the company layout, change the hearing protector and develop a project to optimize the lighting of the factory. In order to comply with labor legislation, there is a need for the preparation of a technical report on environmental conditions of work, formation of an internal accident prevention committee, and development of a medical control and occupational health program, encouraging the practice of periodic exams for employees.
\end{abstract}

Keywords: health; ergonomics; workplace safety; labor legislation.

\section{INTRODUÇÃO}

As indústrias de transformação, apesar de ocuparem posição de destaque em acidentes do trabalho, pela frequência e gravidade dos mesmos, enfrentam resistência na aplicação de recursos para melhoria das condições laborais. Nesse setor estão inclusas as indústrias de produtos madeireiros, que mesmo tendo grande importância no cenário econômico nacional, ainda submetem condições adversas de trabalho aos seus colaboradores.

Embora o desenvolvimento de tecnologias de produção traga novos materiais, a madeira e seus derivados continuam a ser usados em larga escala e em importantes pontos do cotidiano nas sociedades atuais (MARTINS; VIEIRA, 2004). $\mathrm{O}$ processamento mecânico da madeira vem evoluindo ao longo dos anos, desde o processo manual e primitivo até a indústria moderna. $\mathrm{E}$ com o processo de modernização e industrialização do setor madeireiro, surge uma série de novos riscos ocupacionais relacionados a essas atividades.

As atividades nas indústrias madeireiras geram impactos, tanto na natureza quanto na qualidade de vida, causando riscos físicos, químicos, biológicos e ergonômicos. Cerqueira;
Freitas (2013) ressaltaram que a forma de trabalho, associada com ferramentas e máquinas utilizadas no segmento de serrarias, proporciona a realização de atividades com sobrecarga física e com riscos biomecânicos iminentes, que, aliados a problemas ambientais, como ruídos, fuligens e baixo grau de instrução do trabalhador, podem contribuir para a ocorrência de acidentes e doenças ocupacionais.

Diversas circunstâncias de trabalho e da vida cotidiana são maléficas à saúde. Doenças do sistema musculoesquelético e psicológicas (dores nas costas e estresse, por exemplo) compõem as mais importantes causas de absenteísmo e de incapacitação ao trabalho (DUL; WzEERDMEESTER, 2012). Essas situações podem estar associadas, por exemplo, a projetos impróprios e o uso errado de equipamentos e das atividades desenvolvidas.

Iida (2005) afirma que os fatores ambientais podem ser fontes de tensão no ambiente de trabalho, ocasionando condições desfavoráveis como excesso de temperatura, frio, ruído, vibração, iluminação inadequada, bem como o excesso de poeira, gases e fuligens. Esses agentes, dependendo da intensidade e exposição, causam desconforto, aumentam os 
riscos de acidentes e podem provocar danos à saúde do trabalhador. Para prevenir e reduzir esses riscos, normas de segurança e medicina do trabalho foram criadas, sendo fundamental o cumprimento destas para garantir a integridade física e saúde dos trabalhadores.

Nessa perspectiva, o objetivo deste estudo foi avaliar as condições de segurança e salubridade dos postos de trabalho de uma serraria na cidade de Belo Jardim, no agreste pernambucano, e sugerir ações de melhorias que venham a tornar o trabalho mais eficiente e menos desgastante para o trabalhador.

\section{MATERIAL E MÉTODOS}

2.1 Caracterização do local de estudo

O estudo foi desenvolvido numa empresa, chamada de "Empresa A", que atua na industrialização e comercialização de produtos dos setores de madeira e aço, localizada em Belo Jardim, Pernambuco.

Nessa empresa, são beneficiadas madeiras de Pinus taeda e Eucalyptus saligna, oriundas do Paraná e Santa Catarina. O beneficiamento majoritário é de pinus, cerca de $95 \%$ da produção da empresa, que está em processo de transição para retirada do eucalipto.

Fundada em 2002, a "Empresa A" dispõe de cerca de 240 funcionários, dos quais 16 estão alocados no setor de marcenaria, onde operam maquinários para o processamento e beneficiamento da madeira, como fabricação de painéis de média densidade (Medium Density Fiberboard - MDF) revestido, portas internas, rodapés, entre outros.

Os dados foram coletados durante três visitas à empresa, realizadas entre os meses de novembro de 2016 e abril de 2017, nas quais foram observadas as condições de trabalho durante a jornada normal e sem interrupções do processo produtivo. As atividades avaliadas foram referentes à operação de serra circular, tupia, serra circular de bancada, moldureira, serra múltipla, prensa de alta frequência, desengrossadeira e destopadeira (Figura 1). Inicialmente, foram levantados dados quanto à estrutura física da empresa, processo produtivo em geral e produtos finais, documentação de segurança do trabalho e atendimento às normas trabalhistas vigentes. Foram contatados o técnico de segurança do trabalho e o gerente da planta, a fim de disponibilizar essas informações para seguimento do estudo.

Em relação ao setor de segurança do trabalho, o técnico de segurança do trabalho foi questionado sobre a existência de programas de segurança dos colaboradores, como PPRA (Programa de Prevenção de Riscos Ambientais), LTCAT (Laudo Técnico das Condições Ambientais do Trabalho), PCMSO (Programa de Controle Médico e Saúde Ocupacional) e PAE (Plano de Atendimento a Emergências).

Ainda foram discutidas questões como o cumprimento das Normas Regulamentadoras (NRs), aprovadas pela Portaria $\mathrm{n}^{0}$ 3.214, de 1978, e posteriormente alteradas pelo Ministério do Trabalho. Essas normas dispõem sobre os procedimentos obrigatórios de segurança e saúde do trabalhador. Foram avaliadas as NRs aplicáveis ao ramo de atividade da empresa, como NR-4: que estabelece quais profissionais de segurança e medicina do trabalho a empresa deve possuir de acordo com o número de funcionários e o grau de risco da atividade principal; NR-5: que obriga as companhias a instituírem por meio de eleição uma Comissão Interna de Prevenção de Acidentes - CIPA; NR-6: que corrobora a obrigatoriedade da empresa em fornecer os equipamentos de proteção individual
(EPI) adequados a cada risco e treinar os funcionários sobre a maneira correta de usá-los.

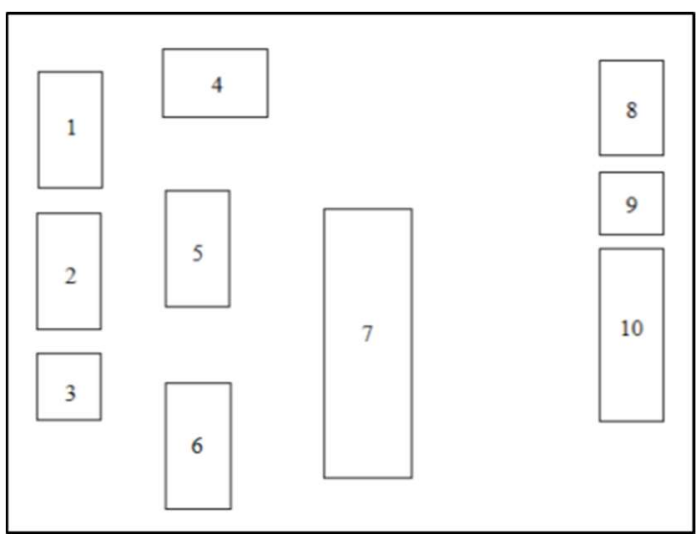

Figura 1. Figura 1. Layout do maquinário do setor de serraria da empresa estudada: 1. Serra circular; 2. Serra circular; 3. Tupia; 4. Serra circular de bancada; 5. Moldureira; 6. Serra múltipla; 7. Prensa de alta frequência; 8. Serra múltipla; 9. Desengrossadeira; 10. Destopadeira.

Figure 1. Layout of the sawmill machinery of the company studied: 1. Circular saw; 2. Circular saw; 3. Tupia; 4. Circular saw bench; 5 . Molding machine; 6 . Multiple sawing; 7 . High-frequency press; 8 . Multiple saw; 9. Thicknesser; 10. Chopping board.

Por fim, foram avaliados qualitativa e quantitativamente os agentes ambientais: ruído contínuo, poeira vegetal respirável, iluminância e calor, com o auxílio de instrumentação específica. Para a avaliação quantitativa desses agentes, foram seguidas as metodologias propostas pelas Normas de Higiene Ocupacional (NHO) da Fundacentro, Normas Regulamentadoras (NRs) do Ministério do Trabalho, e Normas Brasileiras (NBR) da Associação Brasileira de Normas Técnicas (ABNT).

\subsection{Exposição ao ruído contínuo}

$\mathrm{O}$ agente físico ruído contínuo foi avaliado segundo metodologia indicada na NHO-01 (BRASIL, 2001) por meio de medidor de nível de pressão sonora e medidor integrado de uso pessoal, ou apenas dosímetro de ruído.

Para realização da dosimetria de ruído, foi selecionado entre os trabalhadores da serraria, aquele identificado como elemento de maior risco (EMR) para a atividade. O dosímetro de ruído foi posicionado dentro da zona auditiva do trabalhador, mais especificamente no ombro, preso a vestimenta, de forma a fornecer dados representativos da exposição ocupacional. O trabalhador foi avaliado durante todo tempo que ficou de posse do aparelho, cerca de 200 minutos ininterruptos. Os dados obtidos foram processados através do software SONUS do próprio dosímetro.

Aliadas a dosimetria de ruído, foram realizadas dez medições pontuais de ruído em cada uma das máquinas do setor de serraria, através de instrumento de leitura instantânea. Depois de obtidos os resultados através dos relatórios da dosimetria e das leituras instantâneas, os valores foram confrontados com os limites de tolerância instituídos pelo Anexo I da NR-15 (BRASIL, 2015), subtraindo-se o efeito do EPI que o trabalhador utiliza diariamente.

\subsection{Exposição ao calor}

$\mathrm{O}$ agente físico calor foi avaliado seguindo a metodologia da NHO-06 (BRASIL, 2017) por meio do conjunto convencional para determinação de Índice de Bulbo Úmido e 
Termômetro de Globo (IBUTG), que é composto por termômetro de globo - destinado a medição de temperatura de globo (tg) -, termômetro de bulbo úmido natural - destinado a medição de temperatura de bulbo úmido natural (tbn) - e termômetro de bulbo seco - destinado a medição de temperatura de bulbo seco (tbs).

O conjunto foi posicionado com os termômetros alinhados com o plano horizontal, com altura de montagem coincidindo com a área do tórax do trabalhador. Aguardou-se o período de estabilização do sistema, cerca de 30 minutos, e registraramse as leituras dos três termômetros, repetindo a operação por três vezes quando a variação entre as medições foi inferior a $0,2^{\circ} \mathrm{C}$.

Utilizou-se a média dos valores obtidos nas três medições de cada termômetro como resultado final da medição. Registraram-se leituras de temperatura em todos os postos de trabalho, comparando os resultados obtidos com os valores propostos como limite de tolerância pelo Anexo III da NR-15 (BRASIL, 2015).

\subsection{Exposição à poeira vegetal}

$\mathrm{O}$ agente químico poeira vegetal foi avaliado seguindo a metodologia descrita na NHO-08 (BRASIL, 2009), utilizandose uma bomba gravimétrica de fluxo contínuo, devidamente calibrada com vazão regulada em $1,7 \mathrm{~L} \mathrm{~min}^{-1}$, associada a um separador de partículas tipo ciclone de nylon e cassete com filtro de policloreto de vinila (PVC).

O tipo de coleta selecionada para o estudo foi coleta individual, na qual o dispositivo foi colocado no trabalhador, posicionado na altura da zona respiratória. $O$ indivíduo escolhido para a amostra foi o elemento de maior risco (EMR) para a atividade, de acordo com sua proximidade a fonte geradora de particulado e tempo de exposição diária. O tempo de amostragem foi de 410 minutos, $85 \%$ da jornada de trabalho pertinente, utilizando dois amostradores.

Após a coleta do material suspenso no ar, os cassetes foram encaminhados para laboratórios de análises químicas para determinação do teor de poeira vegetal respirável na amostra. A amostragem foi realizada em períodos de tempo diferentes, dessa forma a concentração final resultou do cálculo da concentração ponderada em função do tempo de uso de cada amostrador. Em seguida, os valores resultantes da análise foram confrontados com o limite de tolerância da American Conference of Governmental Industrial Hygienists - ACGIH (2008), visto que a norma brasileira não dispõe sobre poeiras vegetais.

\subsection{Exposição à iluminação}

$\mathrm{O}$ agente iluminância foi avaliado de acordo com a metodologia recomendada pela NBR 5413 (ABNT, 1992) e por meio do Luxímetro Digital, composto por uma célula fotoelétrica, que é posicionada diretamente no campo de trabalho ou a $0,75 \mathrm{~m}$ do piso em um plano horizontal.

Foram realizadas medições de iluminância em todas as máquinas do setor de serraria e os valores obtidos foram comparados com os valores dispostos na NBR 5413 para o tipo de atividade desenvolvida em cada máquina.

\section{RESULTADOS}

3.1. Atendimento às legislações trabalhistas

A "Empresa A" possui dois técnicos de segurança do trabalho, um locado na fábrica e outro itinerante, atendendo as obras. Pela NR-4, de acordo com dimensionamento do Serviço
Especializado de Segurança e Medicina do Trabalho (SESMT), a empresa deveria possuir ainda um Engenheiro de Segurança do Trabalho e um Médico do Trabalho para dar suporte aos colaboradores em jornada parcial, visto o elevado grau de risco da empresa e a quantidade de funcionários.

\subsection{Exposição ao ruído}

O nível de ruído aferido nas dez máquinas do setor de serraria da "Empresa A" encontraram-se acima do limite de tolerância de $85 \mathrm{~dB}$ (A) fixado pela NR-15, sendo a tupia a máquina com maior nível de ruído com $100 \mathrm{~dB}(\mathrm{~A})$ em média, seguida pelas serras circulares com níveis médios de $98 \mathrm{~dB}(\mathrm{~A})$ (Tabela 1). Como resultado da medição dosimétrica da jornada parcial realizada no auxiliar de produção que opera a tupia diariamente, foram obtidos resultados mais elevados (Tabela 2).

Tabela 1. Nível de Pressão Sonora (NPS) médio aferido por máquina. Table 1. Average measured sound pressure level (NPS) per machine.

\begin{tabular}{lc}
\hline \multicolumn{1}{c}{ Máquina/Equipamento } & NPS médio \\
\hline Serra circular & $98 \mathrm{~dB}(\mathrm{~A})$ \\
Serra circular & $97 \mathrm{~dB}(\mathrm{~A})$ \\
Tupia & $100 \mathrm{~dB}(\mathrm{~A})$ \\
Serra circular de bancada & $98 \mathrm{~dB}(\mathrm{~A})$ \\
Moldureira & $94 \mathrm{~dB}(\mathrm{~A})$ \\
Serra múltipla & $93 \mathrm{~dB}(\mathrm{~A})$ \\
Prensa alta frequência & $90 \mathrm{~dB}(\mathrm{~A})$ \\
Serra múltipla & $96 \mathrm{~dB}(\mathrm{~A})$ \\
Desengrossadeira & $91 \mathrm{~dB}(\mathrm{~A})$ \\
Destopadeira & $90 \mathrm{~dB}(\mathrm{~A})$ \\
\hline
\end{tabular}

Tabela 2. Resultados da dosimetria do auxiliar de produção ao manusear a tupia.

Table 2. Results of the dosimetry of the production aid when handling the tupia.

\begin{tabular}{ccc}
\hline Médias & Resultado & Limite de Tolerância \\
\hline LAVG & $109,5 \mathrm{~dB}(\mathrm{~A})$ & $85,0 \mathrm{~dB}(\mathrm{~A})$ \\
NEN & $103,7 \mathrm{~dB}(\mathrm{~A})$ & $85,0 \mathrm{~dB}(\mathrm{~A})$ \\
\hline
\end{tabular}

Nota: LAVG: Average Level - Nível médio de ruído durante o período de análise; NEN: Nível de Exposição Normalizada - Nível de ruído normalizado para uma exposição de 08 horas

\subsection{Exposição ao calor}

Após medição de calor em todas as máquinas do setor verificou-se que em todos os postos de trabalho o IBUTG está abaixo do limite de tolerância proposto pela NR-15 para atividades leves, de pé, em máquina ou bancada, principalmente com os braços, mesmo as análises sendo realizadas na época tradicionalmente mais quente do ano, no mês de dezembro (Tabela 3 ).

Tabela 3. Índice de bulbo úmido e termômetro de globo. Table 3. Globe index and globe thermometer.

\begin{tabular}{lc}
\hline \multicolumn{1}{c}{ Máquina/Equipamento } & IBUTG \\
\hline Serra circular & 26,7 \\
Serra circular & 26,7 \\
Tupia & 26,5 \\
Serra circular de bancada & 26,5 \\
Moldureira & 26,4 \\
Serra múltipla & 26,3 \\
Prensa alta frequência & 26,1 \\
Serra múltipla & 26,3 \\
Desengrossadeira & 26,1 \\
Destopadeira & 26,0 \\
\hline
\end{tabular}




\subsection{Exposição à poeira vegetal}

Com base em uma avaliação qualitativa das máquinas do setor, identificou-se que as serras são os equipamentos que mais produzem poeira, dentre as dez máquinas analisadas. As duas serras circulares e a serra de bancada foram julgadas pelo critério de frequência de uso, para instalação do equipamento que faria a medição quantitativa.

Desta forma, a bomba de amostragem foi posicionada no operador da serra de bancada, visto que a operação da mesma se dava de maneira contínua durante toda a jornada de trabalho, enquanto as serras circulares tinham seu uso intermitente, para trabalhos mais pontuais. Os resultados obtidos encontram-se na Tabela 4.

Tabela 4. Concentração de poeira vegetal.

Table 4. Concentration of vegetable dust.

\begin{tabular}{ccc}
\hline Amostrador & Concentração & Tempo de coleta \\
\hline 1 & $1,652 \mathrm{mg} \mathrm{m}^{-3}$ & 215 minutos \\
2 & $0,855 \mathrm{mg} \mathrm{m}^{-3}$ & 195 minutos \\
\hline Concentração média & $1,273 \mathrm{mg} \mathrm{m}^{-3}$ & 410 minutos \\
\hline
\end{tabular}

\subsection{Exposição à iluminação}

Para tarefas com requisitos visuais limitados e trabalho bruto de maquinaria, a NBR 5413 estipula uma iluminação mínima de 500 lux, englobando assim todas as atividades desenvolvidas no setor que beneficia a madeira. Nenhuma das máquinas analisadas atende as exigências da NBR, estando bem abaixo do limite proposto (Tabela 5).

Tabela 5. Iluminância pelo luxímetro aferido por máquina. Table 5. Machine-gauged luminometer illuminance.

\begin{tabular}{lc}
\hline \multicolumn{1}{c}{ Máquina/Equipamento } & Lux \\
\hline Serra circular & 58 \\
Serra circular & 40 \\
Tupia & 15 \\
Serra circular de bancada & 70 \\
Moldureira & 37 \\
Serra múltipla & 220 \\
Prensa alta frequência & 140 \\
Serra múltipla & 30 \\
Desengrossadeira & 60 \\
Destopadeira & 110 \\
\hline
\end{tabular}

\section{DISCUSSÃO}

Em relação a NR-05, que exige a eleição e formação de uma CIPA anualmente, a "Empresa A", até o mês de março de 2017, estava sem eleger CIPA desde 2014. Além disso, em virtude dos riscos existentes na fábrica, a empresa deveria disponibilizar uma série de treinamentos normativos aos seus funcionários anualmente, como NR-05, NR-06, NR-10, NR11, NR-12, NR-17, NR-18, NR-23 e NR-35. Destes treinamentos, apenas NR-18 e NR-35 são oferecidos para os trabalhadores que realizam a montagem de estruturas de aço para os clientes. Também não há formação de brigada de emergência para atender as ocorrências dentro da fábrica, nem rede de hidrantes instalada, existindo apenas extintores de incêndio, caso ocorra algum princípio de incêndio nas instalações, e os mesmos encontram-se vencidos, obstruídos e sem sinalização, além dos funcionários não possuírem capacitação para manuseá-los.

Quanto ao PCMSO, disposto pela NR-07, a empresa possui o programa médico de seus funcionários, com registro de exames admissional e demissional apenas, não havendo evidências de realização de exames periódicos específicos, como audiometria nos colaboradores expostos ao ruído.

Outro programa de segurança existente na empresa é o PPRA, entretanto, no mesmo só são evidenciadas as fases de antecipação e reconhecimento dos riscos, não havendo medidas para avaliar e controlar os mesmos.

Sobre o fornecimento e uso dos EPIs, a empresa fornece todos os equipamentos e exige o uso por parte dos colaboradores, mas não os treinam sobre a forma correta de usá-los. Os colaboradores, em sua maioria, fazem uso dos EPIs fornecidos pela empresa e disseram estar acostumados a usar e que não se sentem incomodados.

Além do uso, outro ponto importante a ser considerado em relação ao EPI é se ele está sendo efetivo, ou seja, se está reduzindo a intensidade do agente ambiental à níveis que não causam danos aos colaboradores.

De acordo com o Anexo I da NR-15, um indivíduo só poderia se expor ao nível de $103,7 \mathrm{~dB}$ (A) por pouco mais de 30 minutos diários, sem uso de proteção auditiva. No presente estudo, o operador analisado faz uso de um protetor auricular tipo plug, fornecido pela empresa. O EPI é inserido dentro do canal auditivo, e conforme o certificado de aprovação (CA: 5745) no Ministério do Trabalho e Emprego - MTE, ele tem nível de atenuação igual a $18 \mathrm{~dB}(\mathrm{~A})$, se usado da maneira correta.

Como na empresa não foram encontradas evidências de treinamentos do uso correto dos EPIs, não foi possível assegurar se o EPI cumpre de fato sua função atenuando o total de $18 \mathrm{~dB}$ (A). Em contrapartida, essa atenuação do protetor auricular utilizado não é suficiente para tornar salubre a exposição do trabalhador, ou seja, abaixo dos $85 \mathrm{~dB}$ (A) que a norma exige, visto que para uma exposição de 103,7 dB (A), o EPI deveria atenuar no mínimo $18,7 \mathrm{~dB}(\mathrm{~A})$.

Souza et al. (2014) avaliaram as condições de segurança do trabalho em uma serraria no Pará e encontraram resultados semelhantes aos desse estudo, no qual a maioria dos postos de trabalhos apresentou níveis de ruído acima do permitido pela legislação, com valores variando entre 93 e 97 dB (A).

Dessa forma, uma alternativa seria trocar o EPI por um que atenue níveis mais altos, no caso um EPI com atenuação maior que $24 \mathrm{~dB}$ (A) já seria suficiente para minimizar o ruído a níveis abaixo do tolerável. Para os demais funcionários, expostos a níveis abaixo de $103 \mathrm{~dB}(\mathrm{~A})$, o EPI atual se mostra eficiente, atenuando, na maioria dos casos, para abaixo do nível de ação de $80 \mathrm{~dB}(\mathrm{~A})$.

Quanto à localização da tupia, a alteração de sua posição para um local mais distante dos outros equipamentos e, consequentemente, dos operadores, seria uma medida que poderia promover melhorias para os demais indivíduos que estão situados nas proximidades da máquina atualmente, sendo acometidos pelo ruído de fundo produzido pela máquina.

Em relação à temperatura, o Anexo III da NR-15 estabelece como limite de tolerância para trabalho leve e contínuo, ou seja, trabalho sem descanso em local com temperatura mais amena, um índice de até 30,0. Desta forma, recomenda-se à "Empresa A" a instalação de bebedouro no setor, para promover e facilitar a hidratação dos funcionários, e estudar alternativas para melhorar a ventilação como aberturas laterais ou implantação de ventiladores, a fim de melhorar o conforto térmico em dias mais quentes.

A NR-15 não dispõe sobre poeira vegetal, sendo assim, o limite de tolerância utilizado para comparação dos resultados foi obtido da ACGIH, norma americana mais completa que a 
norma brasileira e que passa por constantes atualizações. A ACGIH estabelece o limite de $3,0 \mathrm{mg} \mathrm{m}^{-3}$ para poeira respirável, sendo necessário uma redução (fator de redução $=$ 0,88), pois a jornada semanal de trabalho dos EUA é de 48 horas semanais, enquanto no Brasil a jornada é de 44 horas semanais. Nesse estudo usamos o limite corrigido de 2,64 mg $\mathrm{m}^{-3}$.

Foi observado que o operador usa equipamento de proteção individual durante todo período que se expõe à poeira, mesmo não sendo obrigatório do ponto de vista legal, visto que a concentração do agente foi menor que o limite de ação, que é de $50 \%$ do limite de tolerância. Entretanto, não conhecendo a suscetibilidade do indivíduo, o uso da máscara é aconselhável para, além de evitar o incômodo da poeira da madeira nas vias aéreas, prevenir o operador das doenças causadas pela inalação do material particulado de maneira crônica.

Devido ao risco inerente da operação de máquinas cortantes, a iluminação se mostra um ponto crítico em relação a segurança dos colaboradores. As máquinas que apresentaram valores mais elevados são as que se encontram próximas ao portão, que gera uma grande entrada de luz natural, pois permanece aberto durante todo o dia, entretanto, não é suficiente para iluminar de maneira eficiente todo o pátio da fábrica.

As luminárias que se encontram no local, num total de 8 refletores, não se mostraram eficientes, necessitando de trocar as lâmpadas e fazer a limpeza do aparato em torno. A implantação de mais luminárias, aberturas laterais e substituição de algumas telhas por telhas translúcidas podem gerar uma melhoria no conforto óptico e na segurança dos colaboradores, apesar dos funcionários, quando questionados sobre a iluminação, não realizarem queixas.

\section{CONCLUSÕES}

A "Empresa A" não se adequa totalmente à legislação atual em relação à salubridade e segurança do trabalho, precisando de alguns ajustes. Como recomendações para a "Empresa A", propõem-se:

i) Realizar a troca do equipamento de proteção individual auricular fornecido aos colaboradores, para que possa atenuar os níveis de ruído para abaixo de $85 \mathrm{~dB}$ (A). Também pode ser feita uma mudança no layout do maquinário, além de instituir um rodízio dos funcionários nas máquinas.

ii) Implantar um sistema de iluminação mais eficiente e promover maior ventilação dentro do pátio da fábrica.

iii) Capacitar uma brigada de emergência e uma Comissão Interna de Prevenção de Acidentes, e desenvolver um programa médico completo com apontamento de exames específicos para as atividades da empresa, a fim de monitorar a saúde de seus colaboradores.

iv) Atualizar o Programa de Prevenção de Riscos Ambientais anualmente e inserir as fases de avaliação, com medições quantitativas dos agentes ambientais e controle, evidenciando quais ações serão tomadas durante o ano de vigência do documento, com objetivo de minimizar os riscos encontrados.

Ademais, ressalta-se a importância das empresas, além de fornecerem condições e equipamentos de segurança, instruírem aos funcionários acerca do risco que eles se expõem, por meio de diálogo e realização de treinamentos com todos os trabalhadores, pois, deste modo, as normas de segurança e medicina do trabalho serão, de fato, efetivas.
6. REFERÊNCIAS

ABNT_ASSOCIAÇÃO BRASILEIRA DE NORMAS TÉCEICAS. NBR 5413: Iluminância de Interiores. Rio de Janeiro, 1992. $13 \mathrm{p}$.

ACGIH_AMERICAN CONFERENCE OF GOVEERNMENTAL INDUSTRIAL HYGIENISTS. 2008 TLVs and BEIs Handbook. Cincinnati: ACGIH. 2008. $274 \mathrm{p}$.

BRASIL_MINISTÉRIO DO TRABALHO E EMPREGO. NHO 01: Procedimento Técnico - Avaliação da Exposição Ocupacional ao Ruído. Brasília: Ministério do Trabalho e Emprego. Fundacentro. 2001. 41 p.

BRASIL_Ministério do Trabalho e Emprego. NHO 06: Avaliação da exposição ocupacional ao calor. Brasília: Ministério do Trabalho e Emprego. Fundacentro. 2017. 49 p.

BRASIL_MINISTÉRIO DO TRABALHO E EMPREGO. NHO 08: Coleta de Material Particulado Sólido Suspenso no Ar de Ambientes de Trabalho. Brasília: Ministério do Trabalho e Emprego. Fundacentro. 2009. 24 p.

BRASIL_MINISTÉRIO DO TRABALHO E EMPREGO. NR 15: Atividade e operações insalubres. Ministério do Trabalho e Emprego. 2015.

CERQUEIRA, P. H. A.; FREITAS, L. C. Avaliação da capacidade de trabalho e do perfil de trabalhadores em serrarias no município de Eunápolis, BA. Floresta, Curitiba, v. 43, n. 1, p. 19 - 26, 2013. DOI: http://dx.doi.org/10.5380/rf.v43i1.26021

DUL, J.; WEERDMEESTER, B. Ergonomia prática. 3. ed. São Paulo: Blucher, 2012. 164 p.

IIDA, I. Ergonomia: projeto e produção. 2 ed. São Paulo: Edgard Blucher, 2005. 360 p.

MARTINS, J. G.; VIEIRA, A. Derivados de madeira: série materiais. 1. ed. 2004. 153 p. Disponível em: < https://pt.scribd.com/document/120789512/Materiais-deconstrucao> Acesso em: 28 de jun 2019

SOUZA, D. V.; ALVES, S. A.; REIS, A. R. S.; FREITAS, A. D. D.; MENDES, F. S. Diagnóstico das condições de trabalho em serraria, Uruará, Pará, Brasil. Enciclopédia Biosfera, Goiânia, v. 10, n. 19, p. 2879-2887, 2014. 